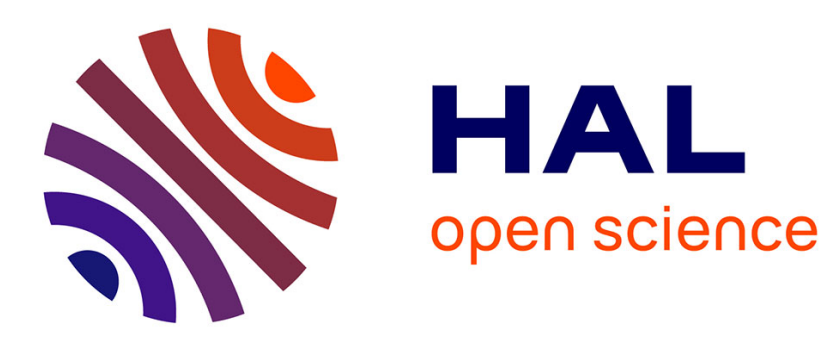

\title{
Toward Efficient Simulation Platform for Platoon Communication in Large Scale C-ITS Scenarios
}

\author{
Ahmed Soua, Oyunchimeg Shagdar, Jean-Marc Lasgouttes
}

\section{To cite this version:}

Ahmed Soua, Oyunchimeg Shagdar, Jean-Marc Lasgouttes. Toward Efficient Simulation Platform for Platoon Communication in Large Scale C-ITS Scenarios. IEEE International Symposium on Networks, Computers and Communications, Jun 2018, Roma, Italy. hal-01878153

\section{HAL Id: hal-01878153 \\ https://hal.inria.fr/hal-01878153}

Submitted on 20 Sep 2018

HAL is a multi-disciplinary open access archive for the deposit and dissemination of scientific research documents, whether they are published or not. The documents may come from teaching and research institutions in France or abroad, or from public or private research centers.
L'archive ouverte pluridisciplinaire HAL, est destinée au dépôt et à la diffusion de documents scientifiques de niveau recherche, publiés ou non, émanant des établissements d'enseignement et de recherche français ou étrangers, des laboratoires publics ou privés. 


\title{
Toward Efficient Simulation Platform for Platoon Communication in Large Scale C-ITS Scenarios
}

\author{
Ahmed Soua*†, Oyunchimeg Shagdar*†, Jean-Marc Lasgouttes* \\ *Inria, 2 Rue Simone Iff, 75012 Paris, France \\ Email: jean-marc.lasgouttes@inria.fr \\ ${ }^{\dagger}$ Institute VEDECOM, 77 Rue des Chantiers, 78000 Versailles, France \\ Email: \{ahmed.soua, oyunchimeg.shagdar\}@ vedecom.fr
}

\begin{abstract}
With recent technological advances made in the field of vehicular communications, a plethora of applications has emerged that aim to improve driver safety and traffic fluidity. The platooning application is expected to be a good first step to tame autonomous driving, especially traffic congestion problems in big cities. In this context, it is important to study a platoon of vehicles in its environment, sharing the road and wireless channel with other road users. For such a scenario, efficient simulation tools come in handy for studying vehicular communications in large scale environments. This paper presents a step toward improving iTETRIS simulation platform to simulate platoon communications in large-scale connected vehicles use case. To this end, a restricted communication zone, centered around the leading vehicle of the platoon, and in which vehicles can receive and send packets is introduced to limit the computational cost of communication simulation. The merits of the improved platform in term of time execution efficiency compared to iTETRIS are shown through extensive simulation using real mobility traces.

Index Terms-Platoon communication; simulation platform; large scale scenario; C-ITS.
\end{abstract}

\section{INTRODUCTION}

Cooperative vehicular communications, based on wireless data exchange between moving vehicles as well as with Road Side Units, have attracted the interest of both industrial and research communities, thanks to their potential capacity to increase road safety and improve traffic efficiency. In fact, these emerging Cooperative Intelligent Transportation Systems (C-ITS) are promising safer roads by enabling several safety applications like lane-changing alert, accident-ahead notification and bad weather conditions alarm. On the other hand, different traffic management-related scenarios have been investigated by these cooperative networks, like lane-merging assistance, parking availability, and platoon communication. This latter use case is regarded as one of the most efficient remedies to congested roads issue in big cities. Vehicle platooning, where vehicles drive in a convoy with small relative distance, is expected to provide higher throughput, improved traffic safety [1][2] and lower energy consumption due to slipstream [3].

Platooning in general is realized through two functioning modes. The first one is based on Adaptive Cruise Control (ACC) [4] mechanism, which uses sensors such as radar, camera and laser to make an estimation of the relative distance and

978-1-5386-3779-1/18/\$31.00 (C)2018 IEEE

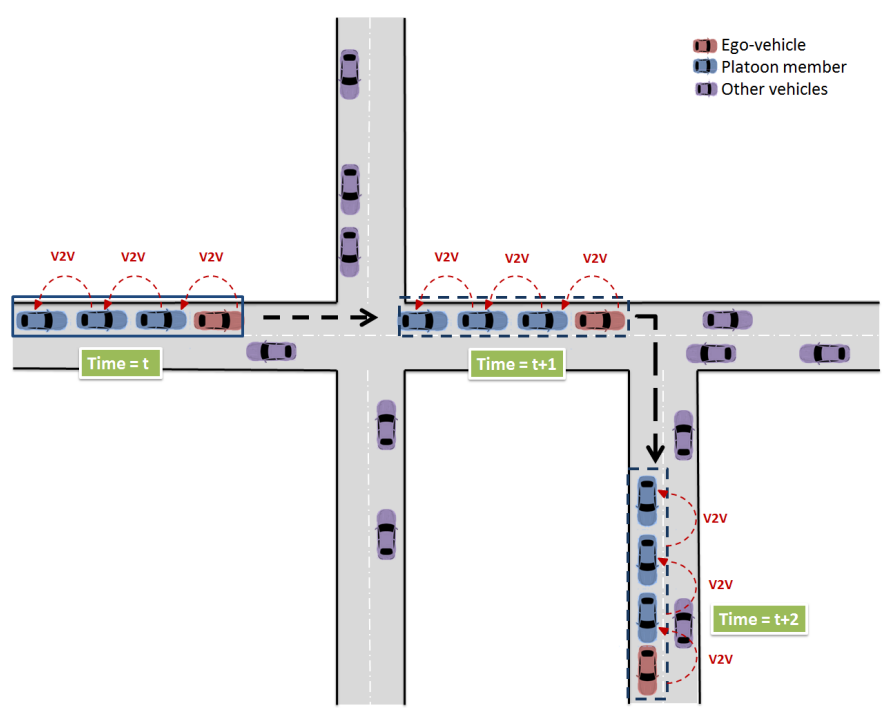

Fig. 1. Cooperative platoon in large scale environment

velocity of two consecutive vehicles. Since ACC mode can not provide drivers with a very precise estimation of the distance gap or the velocity of vehicles, Cooperative Adaptive Cruise Control (CACC) [5] was proposed to tackle these limitations. In this cooperative mode, vehicles in the platoon exchange their dynamic state information like speed, position and heading using Vehicle to Vehicle (V2V) communications as well as Visible Light Communication (VLC) [6]. This information, together with data gathered from on-board sensors, is used to adapt their own acceleration and speed to maintain the inter-vehicle distance at a desired value as shown in Fig. 1.

Most of the existing efforts on platooning have been concentrated on the control/behavior of the individual platoon members [3][4], or on the interaction/communication between the platoon members [6][7] for a stable chain [4][5][7]. Nevertheless, there is a lack of studies that have investigated the platoon in its environment, sharing the road with other vehicles under the strong traffic regulations (traffic lights, speed limits, and so on).

For the latter case, in order to validate its efficiency in alleviating the burden of traffic congestion, platoon communi- 
cation needs to be tested in a real scenario where the convoy of vehicles makes its journey through a large urban area with all its components such as moving vehicles, infrastructures, buildings, roads, radars, etc. Relying on experimental testbeds and real deployment is usually impractical since it requires an access to a large number of fleets and thousands of vehicles equipped with different technologies and communication abilities. For these reasons, simulation tools are regarded as an efficient technique to emulate large scale environments and accommodate their complexities with less technical and financial challenges.

While platooning control and interactions between the platoon members can be simulated using Matlab/Simulink [7] or ProSiVIC [6], these tools cannot be used to simulate a platoon in its environment, where traffic behavior and $\mathrm{V} 2 \mathrm{X}$ communication of a large number of vehicles need to be investigated.

In this paper, we consider the idea of enhancing the performance of iTETRIS [8], a dedicated simulation framework for vehicular communication, in order to tackle very large simulation scenarios. In particular, we present a new technique to adapt iTETRIS to a CACC-based platoon scenario in a city-wide context. iTETRIS consists of two main components: the network simulator ns-3 [9] and the mobility simulator SUMO [10]. The bottleneck is the execution time of ns-3, which is much more processing computation-intensive than SUMO. While ns-3 is able to model wireless networks with a high level of details, it has strong scalability limitations and struggles to simulate networks with only few thousands of nodes. As shown in [11], to process a simulation step of 1 second with 300 nodes sending 1 beacon per second, ns-3 requires approximately 75 seconds of real time. Consequently, a one minute simulation would take several hours to be carried out.

Our solution is based on constructing a specific zone called Restricted Zone (RZone) around the leader of the platoon and letting only the vehicles inside this zone exchange messages. The rest of vehicles are deactivated, since we are only interested on the platoon communication. The key idea behind this restriction is that vehicles situated beyond the interference zone of the platoon leader, are not concerned by the exchanged information between platoon members and not able also to interfere with these communications. It is thus possible to focus only on vehicles located inside the interference zone of the platoon leader.

The rest of this paper is organized as follows: Section II describes some of the state of the art in relation to ITS simulation platforms. In Section III, we present the simulation platform iTETRIS and we highlight its limitations to perform large scale simulations. Then, in Section III-D, we describe the key components of our proposed technique aiming at simulating cooperative platoon communications in large scale environments with low processing time requirements. Section IV investigates the potential of the proposed platform and its efficiency compared to iTETRIS. Conclusion and future work are outlined in Section V.

\section{State of The ART}

Before going in depth with our proposed enhancement to the iTETRIS platform for large scale simulation scenarios, it is worth taking an overview at the available literature on simulation platforms dedicated to cooperative transportation systems.

Usually, simulation tools dedicated to vehicular ad hoc networks (VANETs) are formed of two categories of simulators: networking and traffic simulators. These two components are dependent because, as vehicles move in the traffic platform, the network topology (as wireless nodes) in the networking simulator changes dynamically. While the traffic simulator is used to reproduce realistic vehicular mobility traces in accordance to the surrounding environments, like road topology, traffic lights, human interactions, etc., the networking simulator aims to perform detailed packet-level simulation of source, destinations, data traffic transmission, reception, background load, route, links, and channels [12].

Recent years have witnessed a growing interest in developing more realistic environments to validate ITS applications. Shawn [13], Veins [14] and iTETRIS [8] are examples of simulation platforms that couple a mobility simulator with a network one: while SUMO is the common traffic simulator chosen by all the mentioned platforms, the network simulator is different for each tool. In fact, Shawn integrates its own specific network simulator, while Veins and iTETRIS are using OMNET [15] and ns-3 respectively. This coupling between the two simulators in such platforms, called federated architecture, allows to realistically analyze the influence of VANET-based applications on the road traffic and vice versa.

Another strategy to build VANET simulators is to conceive a unique tool that integrates both a network simulator and a traffic generator. NCTUns [16] stands as a relevant example of this technique. This simulator actually was at first developed as a network simulator, but then integrated some traffic simulation capabilities. NCTUns offers the possibility to simulate various protocols used in both wireless and wired networks and it uses Linux TCP/IP protocol stack to generate high-fidelity simulation results. In this simulator, since the code for the vehicles movement is integrated with the network simulation code, making some extension to the simulator is a very difficult task.

Since this current work is funded by SINETIC project [17], which aims at developing a complete framework to simulate C-ITS based on iTETRIS platform, we focus in this paper on enhancing iTETRIS to perform large scale simulations for platoon application.

\section{SySTEM MODEL}

In this section, the system model of the enhanced platform is presented. We first describe in details the iTETRIS platform and its components, followed by presenting the cooperative platoon application. Then, we highlight our proposed technique to efficiently simulate platoons in large cities aiming at minimizing the processing time of simulations. 


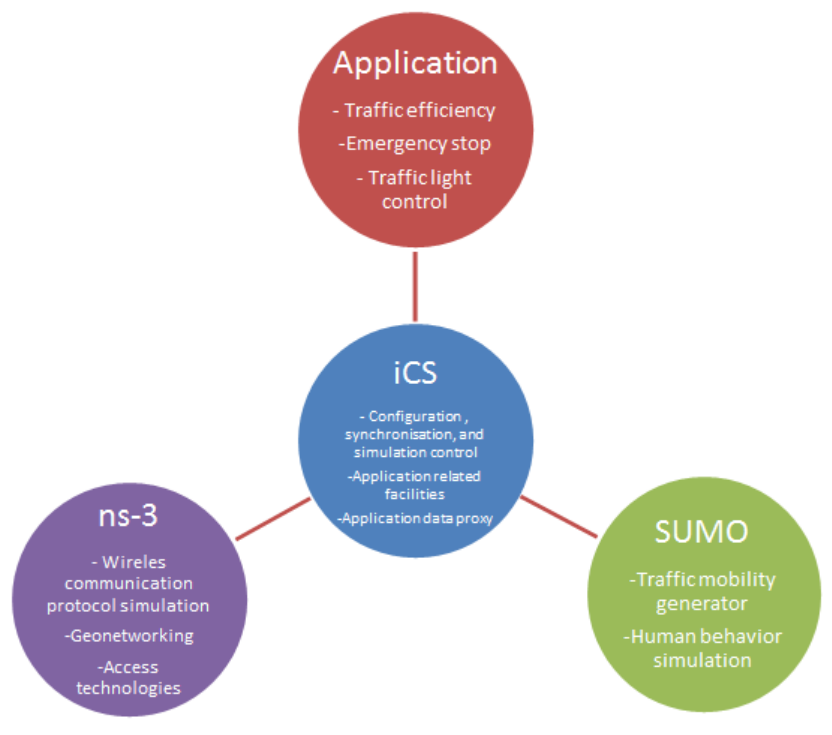

Fig. 2. iTETRIS: Architecture overview

\section{A. The iTETRIS Platform}

We now describe the main components of the iTETRIS simulation framework. A general overview of the the platform is shown in Fig. 2.

The main objective of the platform is to efficiently simulate cooperative vehicular communication with real mobility patterns. The iCS interface is the component responsible for federating the traffic simulator SUMO, the network simulator ns-3 and the implemented ITS applications. In the following, we detail the different elements of this platform.

- SUMO is an open source, microscopic road traffic simulation package designed to handle large road networks. It offers a remote control interface called TraCI that allows to externally adapt the simulation and to control vehicles on the fly. Besides, SUMO incorporates other applications for converting different demand data into a format that the simulation can handle by iTETRIS.

- ns-3 is a scalable network simulator that benefits from a continuous maintenance and rapid growth due to its large development community. It supports a large number of protocols and access technologies, notably the standardized communication protocol dedicated to VANETs IEEE 802.11p. Nevertheless, ns-3 still lacks significant functionality for the close-to-reality modeling and simulation of ITS application. To bridge this gap, the iTETRIS platform adds an ITS-specific communication stack to enable different services like geographic routing, local dynamic map to track relevant messages, and delaytolerant network services.

- iCS is responsible of handling the interactions of ITS applications with both ns-3 and SUMO. It controls the simulation by keeping all simulators synchronized and by triggering actions in different modules. Beyond being a synchronization and data exchange interface between modules, iCS adds specific modules to ns-3 and SUMO to enable the evaluation of ITS applications.

\section{B. Platoon Communication Model}

In this section, we specify the characteristics of the platoon system we want to simulate for the enhanced simulation platform. We suppose that the platoon system uses the CACC mode [5] to maintain its stability, which means that each vehicle in the platoon is equipped with a wireless interface and is able to exchange vehicular status (e.g., speed, orientation, acceleration) with other nodes as depicted by Fig. 1. The ETSI standardized Cooperative Awareness Message (CAM) [18] can be used for intra-platoon communication to maintain a stable platoon, as well as for communication between the platoon and other non-platoon vehicles for collision avoidance [7]. Specifically, each member of the platoon announces its motion to other cars through CAMs. CAMs contain information about the sender like vehicle type, position, heading, velocity. The receiver of such messages interprets them and then adapts its velocity to keep the inter-distance from the preceding vehicle stable. As mentioned earlier, non-platoon vehicles on the road also send CAMs for the purpose of cooperative awareness.

A preliminary implementation of the platoon model inside iTETRIS platform was carried out within SUMO simulator. The main goal behind this implementation is to investigate how we can make iTETRIS faster at simulating platoon communication in the case of city-wide scenario.

\section{Problem Statement}

In this paper, we focus on CACC-enabled platoons where vehicles exchange periodic information to keep the platoon stable (and thus avoid the accordion effect) using iTETRIS platform. In CACC mode, CAMs are broadcast periodically, e.g. every $100 \mathrm{~ms}$, using dedicated short range communication devices (DSRC) [19]. The networking load resulting from several platoons and non-platoon vehicles present in the simulation can lead to packet loss that can degrade the performance of CACC mode. This is the kind of effect that can be studied with a simulator like iTETRIS.

In addition, as stated above, we focus in this work on large scale simulation scenarios, where the total number of vehicles can reach easily thousands of nodes and the area to simulate hundred of $\mathrm{km}^{2}$. The complexity of such scenarios, coupled with the huge amount of CAM transmission, can only make the burden more overwhelming. The simulation of platoon communications in parallel with thousands of normal nodes becomes then a very challenging task.

Furthermore, in order to determine which nodes will receive a transmitted packet for a simulation of $n$ nodes sharing the same wireless channel, ns-3 stores all the $n$ nodes in a list (in the following, this list will be referred as ns3_list). This entire list has to be checked to locate the nodes within the transmission range of a given sender which leads to $O(n)$ iterations. As vehicular transmissions are usually performed repeatedly and the number of sending vehicles can reach thousands of nodes, this list-checking process can dominate the computation time 
during the simulation and thus severely limit the scalability especially when the total number of vehicles $n$ becomes very large.

As part of the project COLOMBO [20], a simple optimization, where communication with too distant nodes is not simulated, has been proposed. However, it's not efficient enough in our case. We will compare it to our approach in Section IV-B.

In order to mitigate the problem of scalability of iTETRIS and to simulate a platoon in its environment, we propose to enhance the performance of ns-3 by limiting the number of sending and receiving nodes to the ones located in a geographic zone centered around the leader of the platoon. This zone corresponds to the interference zone of the platoon leader. In fact, in the case of platooning communications, only the platoon members and nodes that are in the interference range from the platoon, are concerned by the messages transmitted either by the leader or the followers vehicles to maintain the stability of the platoon. Thus, limiting the number of active nodes in the simulation to the vehicles located in this restricted zone will significantly improve the execution time and memory usage, making it feasible to carry out simulations with thousands of nodes buy focusing only on platoon communications.

\section{Proposed Approach}

Our approach aims to make platoon simulations in large scale systems more efficient in term of execution time. Contrary to conventional vehicular simulation, where researchers are interested on evaluating their communication protocol in a large number of nodes from a bird's eye perspective, we focus in this work on a group of vehicles going in the same direction forming a platoon. The leader of this platoon is referred in the rest of paper as the ego-vehicle.

Platoon applications have specific characteristics that need to be taken into account when designing the new solution to make iTETRIS simulation faster in the case of platoon communications: they focus on the communication inside the platoon members to maintain the stability of the vehicles convoy as well as the possible messages, sent by non-platoon cars, that can interfere with the platoon members. Thus, only platoon members and the vehicles that interfere with the platoon's communication need to be taken into consideration for the wireless transmissions since platoon applications packets are only relevant for these nodes. However, although the contents of the packets sent by vehicles not belonging to the platoon but close enough to interfere with it is not relevant to the platoon application, these messages have to be simulated inside the wireless simulator in order to realistically evaluate wireless resource demands and bandwidth consumption.

Starting from these crucial distinction, we propose to define an area of interest centered around the ego-vehicle and in which the simulation of transmission packets is fulfilled. This area of interest, called Restricted Zone (RZone), moves during the simulation, since it is following the ego-vehicle as it moves through the city. Fig. 3 shows a schematic view of the dynamic restricted zone. This latter is defined as a disk centered around the leader of the platoon (red color). Dark

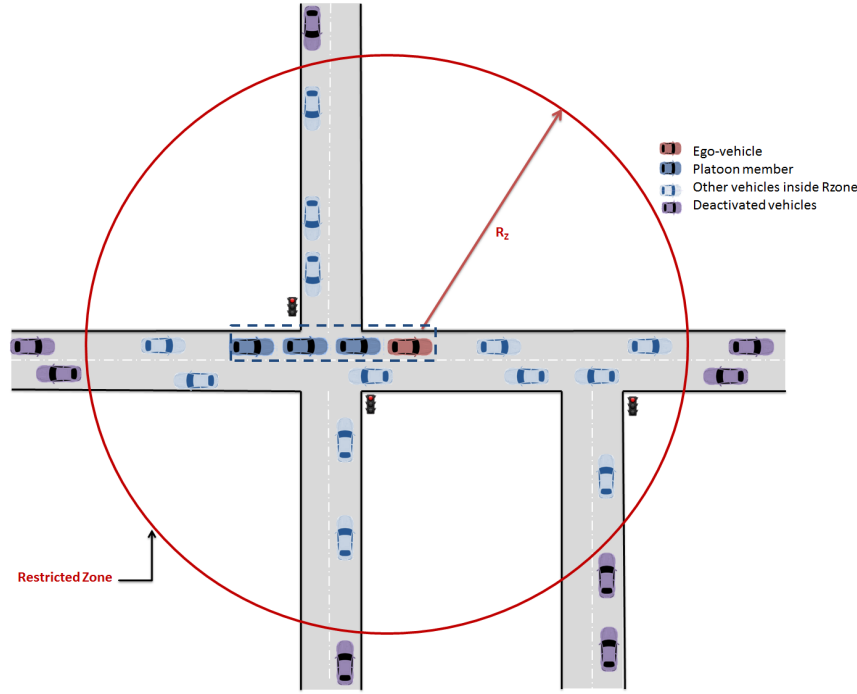

Fig. 3. Restricted Zone of transmission for platoon communications

blue vehicles (members of the platoon) are within that disk and are thus simulated in the wireless simulator ns-3. Other normal vehicles that are inside the zone (sky blue color) can also receive and transmit messages. These nodes, as it is the case in conventional VANETs, are periodically sending CAMs to advertise their positions to the neighboring cars. This is useful to depict the effects of the near surrounding transmissions on the platoon communication. The radius of the Restricted zone, namely $R_{z}$, allows us to prevent vehicles outside the relevant zone (mauve color) to be simulated in ns- 3 . The idea is that these vehicles are too far from the ego-vehicle to interfere with it. Thus, by limiting the number of transmitting/receiving vehicles to only the nodes located inside the restricted zone, the size of ns3_list is much lower compared to "all nodes transmitting" technique and consequently the complexity of calculation dramatically decreases. We have to mention here that our proposal treats the interaction of a specific group of vehicles with its near environment, and then it can not be applied to accurately simulate the communication of a complete city-wide scenario.

While the above description of our proposal to reduce simulation temporal complexity defines a single ego-vehicle and then a single RZone, the concept can be generalized by defining multiple ego-vehicles to simulate a specific number of platoons driving through the city. In this case, a different RZone will be associated to each platoon leader, making our technique able to scale to very large vehicular systems and at the same time reducing simulation time complexity.

\section{Evaluation}

\section{A. Scenario and Simulation Setup}

In this section, we show simulation results and investigate the performances of our proposal in terms of simulation execution time. We compare our scheme to the original version of iTETRIS as well as its improved version COLOMBO. To 
TABLE I

LUXEMBOURG CITY TRAFFIC MODEL

\begin{tabular}{ll}
\hline Area & $155.95 \mathrm{~km}^{2}$ \\
Total nodes & 2500 \\
Total length edges & $931.12 \mathrm{~km}$ \\
Traffic lights & 203 \\
Buildings & 13553 \\
\hline Platoon size & 5 \\
CAM message size & 400 bytes \\
CAM periodicity & $100 \mathrm{~ms}$ \\
Simulated time & $10 \mathrm{~min}$ \\
$R_{z}$ & $2000 \mathrm{~m}$ \\
\hline
\end{tabular}

carry out platoon simulations in a large scale system, the first step is to choose an appropriate traffic scenario that can reproduce realistic mobility patterns. To this end, we have selected the Luxembourg SUMO Traffic (LuST) scenario [21] as a synthetic example, since it provides us with realistic urban traffic traces of a mid-size European city, exhibiting different mobility patterns during the rush hour (high density), the day (moderate density) and the night (low density).

The scenario, as described by Table I, consists in simulating a platoon inside Luxembourg, in an area of $156 \mathrm{~km}^{2}$, with a total length of roads equal to $931 \mathrm{~km}$. The number of cars can reach 2500 during rush hours.

We performed the simulations on a PC equipped with an Intel Core CPU i7-5500U at $2.4 \mathrm{GHZ}$ with $8 \mathrm{~GB}$ of RAM. The operating system is Ubuntu Linux 16.04 with 64 bit kernel. We used the latest version of COLOMBO to develop our restricted zone technique. The ego-vehicle starts in the center of Luxembourg city and starts moving as the simulation is running. The platoon we construct is composed of 4 members and is following the platoon leader during its journey. The radius of the restricted zone is fixed to $2000 \mathrm{~m}$ and is large enough to consider all the vehicles whose transmission can interfere with the communications of the platoon, since transmission range of $\mathrm{V} 2 \mathrm{~V}$ using the IEEE $802.11 \mathrm{p}$ is around $500 \mathrm{~m}$. We simulate 10 min of wireless traffic at different moments of the day. In fact, we are interested on some specific 10 min time slices where the density of vehicles in the simulation is reflecting different traffic situations (low, medium and high density of traffic). We investigate in the following how long these $10 \mathrm{~min}$ of simulated time will require in term of real processing time.

\section{B. Performance Evaluation}

We conducted extensive simulation experiments to measure the duration that the simulation takes to perform $10 \mathrm{~min}$ of wireless packets exchange for our proposal and we compared it to iTETRIS and COLOMBO versions.

Fig. 4 shows the performance of the performed simulations in terms of processing time during different times of the day (with different vehicle densities). We can clearly see that our technique outperforms largely iTETRIS for all the different densities. For example, at 8 am (which corresponds to a high density of vehicles), iTETRIS performs the 10 min simulation

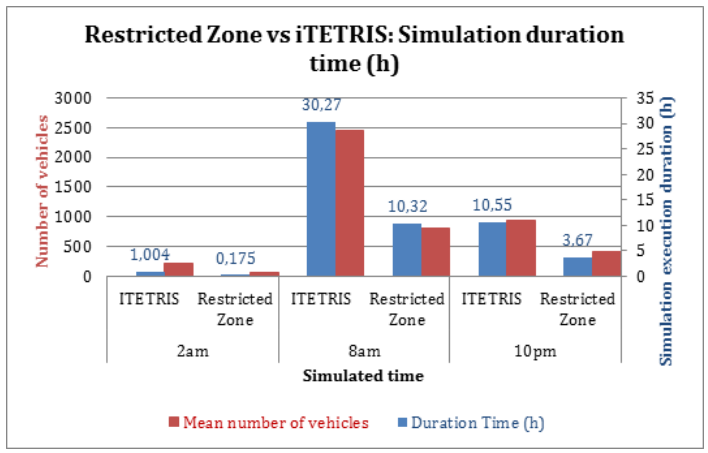

Fig. 4. Simulation execution time comparison: iTETRIS vs Restricted Zone

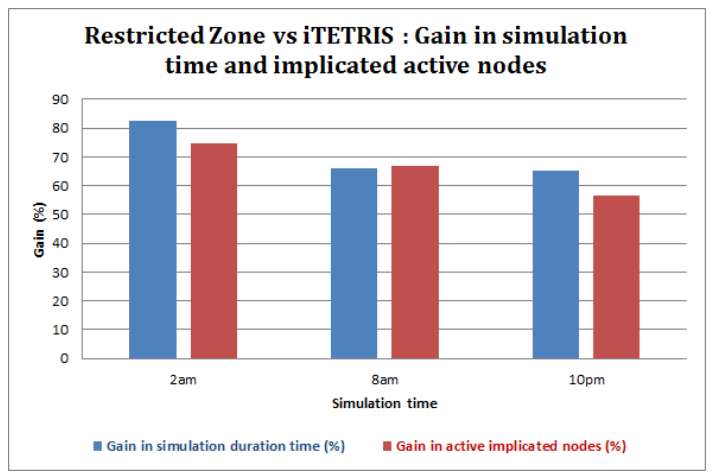

Fig. 5. RZone vs iTETRIS: Gain in simulation execution time and implicated active nodes

in $30.27 \mathrm{~h}$, while our proposal finishes it after only $10.32 \mathrm{~h}$. The same efficiency of the Restricted Zone approach can be noticed also at 10pm, where the densities of nodes is medium. This remarkable gain in term of simulation time is explained by the fact that with iTETRIS, all the nodes present in the simulations are able to transmit and receive packets (like beacons, event driven messages, etc.) in addition to the platoon communications which makes the wireless transmissions load very huge specially with high vehicles densities. On the other hand, using our technique, only cars present in the Restricted Zone are given the right to exchange messages which sharply limits the size of the ns 3 list, reducing accordingly the time processing inside ns-3. This observation is confirmed by Fig. 5, where we compute the gain in simulation time as follows:

$$
\left[1-\frac{\text { SimulationTime }(\text { RZone })}{\text { SimulationTime }(\text { iTETRIS })}\right] \times 100
$$

We can see clearly that our RZone approach maintains a minimum gain of $65 \%$ in term of simulation processing time and for different densities compared to iTETRIS. This gain can reach $82 \%$ in the case of low densities (2am).

Fig. 5 confirms also the observations stated before regarding the number of implicated nodes and how it severely affects the simulation processing. In fact, it demonstrates that RZone can ensure a minimum gain in term of simulated vehicles equal to $56 \%$ and it can be over $70 \%$ at lower densities (2am). This gain 


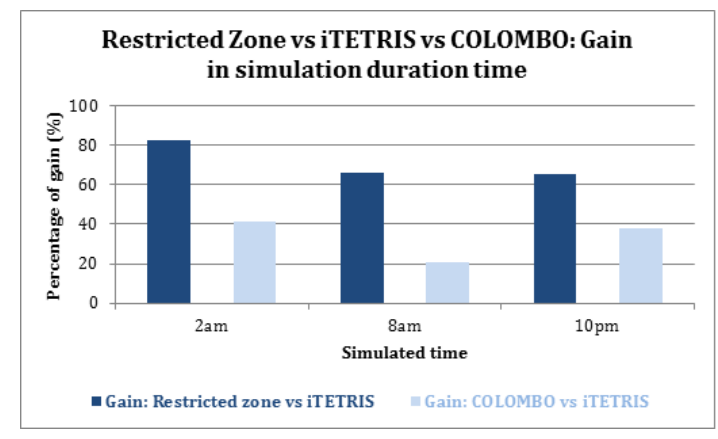

Fig. 6. iTETRIS vs COLOMBO vs RZone: Gain in implicated nodes

is explained by the fact that we limit the wireless transmissions to only the ones inside the circle of radius $R_{z}$.

To highlight the efficiency of our proposal compared to other enhancements made to iTETRIS, we compare in Fig. 6 iTETRIS and COLOMBO (seen as an improvement to iTETRIS) in term of simulation duration time. We observe clearly that even if COLOMBO improves the overall simulation duration time when simulating the platoon, this reduction still smaller than the one introduced by our RZone technique. In fact, COLOMBO ensures a minimum gain of $20 \%$ compared to iTETRIS but can not exceed the value of $40 \%$ in best cases. This can be explained by the fact that COLOMBO removes only the nodes that are very distant (located in the interference zone) from the ns-3 list to check ( $n s 3$ _list) but maintains all the nodes present in the simulation able to communicate and thus the reduction in the total of nodes to simulate is modest compared to iTETRIS.

The above performance evaluations corroborate that the restricted zone approach for platoon communications can spectacularly alleviate the burden of high simulation temporal complexity when dealing with large scale systems and consequently open widely the doors to more efficient simulation platforms for platooning.

\section{Conclusion}

In this paper, we have introduced a concept for making large scale simulations for cooperative platoons more efficient in term of simulation processing time. The presented approach is based on the definition of a dynamic zone of interest called Restricted Zone (RZone), centered around the leader of the platoon and which defines the vehicles that can transmit and receive packets. The performance evaluation of our proposal shows a dramatic reduction of the computation time in comparison to iTETRIS and COLOMBO platforms. This is consistent with the huge reduction of active wireless nodes in ns-3 achieved by limiting the transmission and reception zone to the circle area defined by RZone. As next steps, we are investigating the set up of multiple restricted zones to be able to simulate several vehicular platoons inside the same scenario and then move further towards simulating city-wide scenarios with efficient time processing performances.

\section{ACKNOWLEDGMENTS}

This work benefited from the support of the project SINETIC of the French "Fonds unique interministriel" (FUI).

\section{REFERENCES}

[1] R. Hall and C. Chin, "Vehicle sorting for platoon formation: Impacts on highway entry and throughput," Transp. Res. C, Emerging Technol, vol 12 , no. $5 / 6$, pp. $405-420,2005$.

[2] B. van Arem, C. J. G. van Driel, and R. Visser, "The impact of cooperative adaptive cruise control on traffic-flow characteristics," IEEE Trans.Intell. Transp. Syst., vol. 7, no. 4, pp. 429-436, Dec. 2006.

[3] S. Shladover, "Cooperative (rather than autonomous) vehicle-highway automation systems," Intelligent Transportation Systems Magazine, IEEE, vol. 1 , no. 1 , pp. 10-19, Spring 2009.

[4] S. H. Hosseinnia, I. Tejado, V. Milans, J. Villagr, and B. M. Vinagre, "Experimental application of hybrid fractional-order adaptive cruise control at low speed," IEEE Transactions on Control Systems Technology, vol. 22, no. 6, pp. 2329-2336, Nov 2014.

[5] G. Orosz, "Connected cruise control: modeling, delay effects, and nonlinear behavior," International Journal of Vehicle Mechanics and Mobility: Vehicle System Dynamics, vol. 54, pp. 1147-1176, 2016.

[6] M.Y. Abualhoul, P.Merdrignac, O.Shagdar, F. Nashashibi, "Study and evaluation of laser-based perception and light communication for a platoon of autonomous vehicles," IEEE 19th International Conference on Intelligent Transportation Systems (ITSC), pp. 1798-1804, 2016.

[7] O. Shagdar, F. Nashashibi, S. Tohme, "Performance study of CAM over IEEE $802.11 \mathrm{p}$ for cooperative adaptive cruise control," IEEE Wireless Days, March 2017.

[8] V. Kumar, L. Lin, D. Krajzewicz, F. Hrizi, O. Martinez, J. Gozalvez, R. Bauza, "iTETRIS: adaptation of ITS technologies for large scale integrated simulation," IEEE 71st Vehicular Technology Conference (VTC Spring), 2010.

[9] The ns-3 nework simulator [Online]. Available: https://www.nsnam.org.

[10] D. Krajzewicz, G. Hertkorn, C.Rossel, and P. Wagner, "Sumo (simulation of urban mobility)," IEEE 4th Middle East Symposium on Simulation and Modeling, pages 183-187, 2002.

[11] R. Fernandes, and M. Ferreira, "Scalable VANET simulations with ns-3," IEEE 75th Vehicular Technology Conference (VTC Spring), pp. 1-5, may 2012.

[12] A. Grzybek, M. Seredynski, G. Danoy, "Aspects and trends in realistic VANET simulations," IEEE International Symposium on a World of Wireless, Mobile and Multimedia Networks (WoWMoM), 2012.

[13] D. Pfisterer, "Comprehensive development support for wireless sensor networks," Ph.D. dissertation, Institute of Telematics, University of Luebeck, Germany, 2007.

[14] C. Sommer, R. German, and F. Dressler, "Bidirectionally coupled network and road traffic simulation for improved ivc analysis," IEEE Transactions on Mobile Computing," vol. 10, no. 1, pp. 3-15, Jan 2011.

[15] A. Varga, "The OMNET++ discrete event simulation system," European Simulation Multiconference, pp. 319-324, Prague, Czech Republic, June 2001.

[16] S. Wang, and C. Lin, "Nctuns 5.0: A network simulator for ieee 802.11 (p) and 1609 wireless vehicular network researches," IEEE 68th Vehicular Technology Conference (VTC Fall), pp. 1-2, 2008.

[17] The SINETIC project [Online]. Available: https://team.inria.fr/rits/projet/sinetic.

[18] European Telecommunications Standards Institute (ETSI), "Intelligent Transport Systems (ITS); Vehicular Communications; Basic Set of Applications; Part 2: Specification of Cooperative Awareness Basic Service," 2011.

[19] D. Caveney, "Cooperative vehicular safety applications," IEEE Control Systems Magazine, vol. 30, no. 4, pp. 38-53, 2010.

[20] The COLOMBO Project [Online]. Available: http://www.colombofp7.eu/.

[21] L. Codeca, R. Frank, T. Engel, "Luxembourg SUMO Traffic (LuST) Scenario: 24 Hours of Mobility for Vehicular Networking Research," IEEE Vehicular Networking Conference (VNC), 2015. 